\title{
Effects of Deep Brain Stimulation of the Subthalamic Nucleus Settings on Voice Quality, Intensity, and Prosody in Parkinson's Disease: Preliminary Evidence for Speech Optimization
}

\author{
Anita Abeyesekera, Scott Adams, Cynthia Mancinelli, Thea Knowles, \\ Greydon Gilmore, Mehdi Delrobaei, Mandar Jog
}

\begin{abstract}
Objective: To systematically evaluate how different deep brain stimulation of the subthalamic nucleus (STN-DBS) amplitude, frequency, and pulse-width electrical parameter settings impact speech intensity, voice quality, and prosody of speech in Parkinson's disease (PD). Methods: Ten individuals with PD receiving bilateral STN-DBS treatments were seen for three baseline and five treatment visits. The five treatment visits involved an examination of the standard clinical settings as well as manipulation of different combinations of frequency (low, mid, and high), pulse width (low, mid, and high), and voltage (low, mid, and high) of stimulation. Measures of speech intensity, jitter, shimmer, harmonics-noise ratio, semitone standard deviation, and listener ratings of voice quality and prosody were obtained for each STN-DBS manipulation. Results: The combinations of lower frequency, lower pulse width, and higher voltage settings were associated with improved speech outcomes compared to the current standard clinical settings. In addition, decreased total electrical energy delivered to the STN appears to be associated with speech improvements. Conclusions: This study provides preliminary evidence that STN-DBS may be optimized for Parkinson-related problems with voice quality, speech intensity, and prosody of speech.
\end{abstract}

RÉSUMÉ: Les effets de divers réglages de stimulation cérébrale profonde destinée au noyau sous-thalamique sur la qualité de la voix, sur l'intensité de la parole et sur la prosodie de la parole chez des patients atteints de la maladie de Parkinson. Objectif : Évaluer de façon systématique comment différents niveaux de stimulation cérébrale profonde (SCP) du noyau sous-thalamique peuvent avoir un impact sur l'intensité et la prosodie de la parole de patients atteints de la maladie de Parkinson (MP) de même que sur la qualité de leur voix. Pour ce faire, différents réglages de nature électrique ont été envisagés en ce qui a trait à l'amplitude, la fréquence et la durée des impulsions induites. Méthodes : Au total, 10 patients atteints de MP et bénéficiant de traitements de SCP destinés à leur noyau sous-thalamique ont été vus dans le cadre de 3 examens préliminaires et de 5 visites prévoyant un traitement. Ces 5 visites de nature thérapeutique ont sous-tendu un examen des réglages cliniques standards de même qu'une manipulation permettant de combiner, en matière de SCP, diverses fréquences (basses, moyennes, élevées), diverses durées des impulsions (courtes, moyennes, longues) et diverses tensions (basses, moyennes, élevées). Pour chaque intervention de SCP visant le noyau sous-thalamique, nous avons obtenu des mesures portant sur les aspects suivants : intensité, agitation et tremblement de la parole ; rapport harmoniques/bruits ; écart-type d'un demi-ton ; et finalement des notations d'auditeurs en ce qui regarde la qualité de la voix et la prosodie. Résultats : Le fait de combiner des réglages prévoyant des basses fréquences, des durées d'impulsion courtes et des tensions élevées a été associé, en comparaison avec les réglages cliniques standards actuels, à des résultats améliorés en matière de parole. Qui plus est, une diminution totale de l'énergie électrique destinée au noyau sous-thalamique semble aussi être associée à des résultats améliorés en matière de parole. Conclusions : Cette étude contient donc des preuves préliminaires suggérant que la SCP du noyau sous-thalamique pourrait être optimisée pour traiter des problèmes de qualité de la voix, mais également d'intensité et de prosodie de la parole, qui sont relatifs à la MP.

Keywords: Parkinson's disease, Deep brain stimulation, Motor speech disorders

doi:10.1017/cjn.2019.16

Can J Neurol Sci. 2019; 46: 287-294

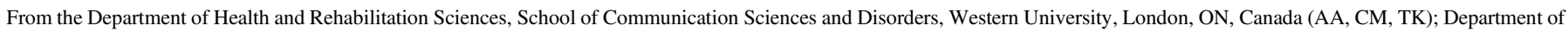

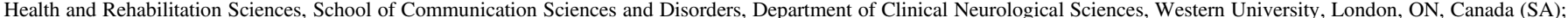

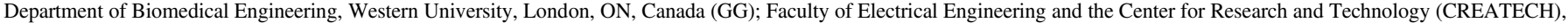
K. N. Toosi University of Technology, Tehran, Iran (MD); Department of Clinical Neurological Sciences, Western University, London, ON, Canada (MJ).

Received August 13, 2018. Final Revisions Submitted January 29, 2019. Date of Acceptance January 31, 2019.

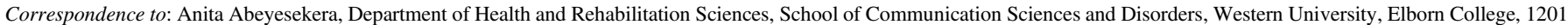

Western Road, London, ON, N6G 1H1, Canada. Email: aabeyese@uwo.ca 


\section{INTRODUCTION}

Idiopathic Parkinson's disease (PD) is associated with speechrelated difficulties such as low speech intensity, abnormal voice quality, and speech that is monoloud and monopitch impacting prosody of speech. ${ }^{1}$ The indirect pathway of the basal gangliathalamocortical circuit is targeted for deep brain stimulation (DBS) in PD patients, as this pathway is related to increased inhibition of movements in PD pathology. ${ }^{2}$ Although deep brain stimulation of the subthalamic nucleus (STN-DBS) is an effective treatment for most major symptoms of $\mathrm{PD},{ }^{3-6}$ effects on speech have been inconsistent across studies. ${ }^{6-21}$

\section{STN-DBS Programming}

The DBS pulse generator enables programming of the voltage or amplitude of the electrical signal (volts/V), the pulse width or the duration of the electrical pulse (microseconds/ $\mu$ s), and the frequency or number of electrical pulses transmitted per second (hertz/Hz). ${ }^{22}$ Given that the value of these three stimulation parameters (voltage, pulse width, and frequency) can be adjusted independently and set to various combinations, there is the potential to identify optimized settings for speech. Standard therapeutic STN-DBS settings generally use 130-180 Hz, 2.5$3.5 \mathrm{~V}$, and $60-90 \mu$ s pulse-width stimulation. ${ }^{23}$ Although success of optimization for most major symptoms of the disease is high, preliminary studies suggest that standard STN-DBS stimulation parameters may not be optimized for the treatment of speech symptoms. ${ }^{6-9}$ Still, few studies have examined manipulations of each parameter setting on speech.

Previous studies highlight the inconsistencies with regard to impact of STN-DBS on voice quality, speech intensity, and prosody; for example, some studies have found improvements in these speech symptoms (voice quality, speech intensity, prosody), worsening of symptoms, and others observing no change. ${ }^{6-21}$ It is important to note, however, that previous study conditions included examination of one parameter setting in isolation, examined standard clinical setting combinations with DBS "on" compared to "off," or did not report specific DBS settings. These studies provide a starting point for programming STN-DBS for speech improvements; however, an important gap in the literature remains. The three stimulation parameters (voltage, pulse width, and frequency) each require programming, and it remains unclear whether different combinations of stimulation settings may result in improved speech outcomes. It is important to examine all potential features of the device. This is particularly true of pulse width and voltage, as relatively few studies have focused on examination of these two parameters. This is also important prior to fully understanding the mechanisms related to speech disorder in PD.

To our knowledge, no previous study has performed a systematic evaluation of a wide range of STN-DBS stimulation parameters on acoustic and perceptual measures of speech in PD.

\section{Purpose}

This STN-DBS study involves a systematic manipulation of different amplitude, frequency, and pulse-width settings on speech production in PD. The purpose of the current study was to provide optimized STN-DBS settings for speech intensity, voice quality, and speech prosody in PD.

\section{Methods}

\section{Participants}

The current study was part of a larger investigation of other motor responses (gait disturbances, bradykinesia, and tremor) to STN-DBS. PD patients' eligibility for STN-DBS surgery included (1) diagnosis of PD with debilitating motor symptoms and (2) severe motor fluctuations including dyskinesia during "on" phases and disabling "off" periods. Exclusion criteria included dementia or severe cognitive impairment as assessed by the Mini-Mental State Examination (cutoff score of 23/30). Exclusionary criterion for participation in the current study included lack of English proficiency. Ten individuals with PD (females; $n=4$ ) who were receiving bilateral STN-DBS treatment served as participants. Twelve participants were recruited, with two participants forced to drop out of the study due to inability to tolerate all of the setting changes and inability to attend all of the scheduled study visits, respectively. Mean age was 63.9 years (range 52-69 years), and mean years since

Table 1: Participant demographics

\begin{tabular}{|c|c|c|c|c|c|c|c|c|}
\hline Patient ID & PD duration & Age & Sex & $\begin{array}{c}\text { MOCA } \\
\text { (pre-operative) }\end{array}$ & $\begin{array}{c}\text { UPDRS }^{\mathbf{b}} \\
\text { (pre-operative) }\end{array}$ & $\begin{array}{c}\text { UPDRS }^{\mathbf{b}} \\
\text { (final visit) }\end{array}$ & $\begin{array}{c}\text { LED }^{\mathbf{a}} \\
\text { (pre-operative) }\end{array}$ & $\begin{array}{c}\text { LED }^{\mathrm{a}} \\
\text { (final visit) }\end{array}$ \\
\hline DBS 01 & 17 & 69 & $\mathrm{~F}$ & 22 & 13.5 & 11 & 2375 & 450 \\
\hline DBS 02 & 10 & 61 & M & 24 & 45 & 12.5 & 1050 & 710 \\
\hline DBS 03 & 11 & 69 & $\mathrm{~F}$ & 27 & 30 & 11 & 750 & 375 \\
\hline DBS 04 & 9 & 64 & $\mathrm{~F}$ & 28 & 20.5 & 17.5 & 1437.5 & 500 \\
\hline DBS 05 & 14 & 65 & M & 26 & 13 & 10 & 1653.75 & 250 \\
\hline DBS 06 & 7 & 67 & M & 23 & 43.5 & 18.5 & 1725 & 200 \\
\hline DBS 07 & 13 & 67 & M & 24 & 28.5 & 4.5 & 1200 & 300 \\
\hline DBS 08 & 10 & 68 & M & 27 & 6.5 & 10 & 1550 & 850 \\
\hline DBS 09 & 6 & 57 & $\mathrm{~F}$ & 25 & 7.5 & 6.5 & 1375 & 0 \\
\hline DBS 10 & 9 & 52 & M & 29 & 13 & 11.5 & 1062.5 & 512.5 \\
\hline
\end{tabular}

${ }^{\mathrm{a}}$ LED was calculated according to Tomlinson et al. ${ }^{24}$

${ }^{\mathrm{b}}$ UPDRS Part III. 
diagnosis was 10.6 years (range 6-17 years). All participants were diagnosed with PD based on Movement Disorder Society (MDS) criteria and recommended to have STN-DBS surgery by neurologist (MD) based on standard clinical treatment protocol for PD. Participant demographics including Unified Parkinson's Disease Rating Scale (UPDRS), Montreal Cognitive Assessment (MOCA), and levodopa equivalent dose (LED) scores are provided in Table 1. PD participants continued with their pharmacological treatment following STN-DBS surgery, with standard medication titration as assessed by the neurologist to avoid withdrawal symptoms and dyskinesia.

\section{Standard Protocol Approvals, Registrations, and Patient Consents}

This study was approved by the Human Subjects Research Ethics Board (HSREB) (Western University Ethics (WUE) No. 103928). Written informed consent was provided by all participants.

\section{Surgical Procedures}

Pre-operative magnetic resonance imaging (MRI) and computed tomography were used to determine the best STN location for target stimulation and entry site. Patients were anesthetized with local anesthesia during implantation and a stereotactic frame was used for burr hole drilling and implantation procedures. Five $60 \mu \mathrm{m}$ diameter tungsten microelectrodes (impedance of 0.5-1.0 $\mathrm{m} \Omega$ at $1 \mathrm{kHz}$; FHC Inc., Bowdoinham, ME) were used to identify STN boundaries. ${ }^{3}$ Microelectrode recordings began $10 \mathrm{~mm}$ above the surgical target and extended 4-5 mm below the target. The microelectrode track that produced the most beneficial motor response to stimulation (alleviation of tremor/rigidity) and fewest side effects (oculomotor, speech) was determined by the neurosurgeons. A chronic therapeutic lead (Model 3389, Medtronic, Minneapolis, MN, USA; $1.5 \mathrm{~mm}$ contact length, 0.5 spacing,
$1.27 \mathrm{~mm}$ diameter) was then permanently implanted into the selected track. The same process was completed bilaterally with fluoroscopy confirmation completed during and after implantation. The Implantable Pulse Generator (Activa PC) for both sides was then implanted subcutaneously into the subclavicular area under general anesthesia. Both monopolar (pulse generator used as the positive contact point) and bipolar settings (contact points for both cathode and anode) were used depending on optimal symptom alleviation as determined by the neurologist during the programming sessions. Post-operative electrode contact location data are presented in Table 2 .

\section{Study Procedures}

Each participant was seen for three baseline and five treatment visits. Baseline visits consisted of the following: (1) pre-operative visit, (2) 1-week post-operative with stimulator "off," and (3) 2-week post-operative with stimulator turned "on" with highfrequency $(130 \mathrm{~Hz})$, mid-pulse-width $(90 \mu \mathrm{s})$, and low-voltage $(1.5 \mathrm{~V})$ settings used. The 5 treatment visits consisted of monthly visits to the laboratory across 6 months during which 24stimulation parameter setting variations were examined. Settings included permutations of three voltage (low, medium, and high), three frequency (low, medium, and high), and three pulse-width (low, medium, and high) settings. Each visit consisted of four sessions during which four stimulation setting combinations were evaluated. These included the participant's standard clinical stimulation settings (programmed by neurologist) and three randomly determined experimental setting combinations. Participants were given between 30 and 60 min to adjust to each experimental setting before testing was resumed. Participants received each experimental stimulation setting combination once throughout the entire study. With the exception of the pre-operative session (Visit 1), participants were tested "off" medication. A speech protocol consisting of 5 speech tasks was administered during each of the 20 sessions (4 sessions at each

Table 2: Localization of active contacts. The position of the active contact was classified as "within the STN," "outside the STN," or "at the interface"

\begin{tabular}{l|c|c|c|c}
\hline Patient ID & Active contact (left) & Left contact position & Active contact (right) & Right contact position \\
\hline DBS 01 & $\mathrm{C}^{+} 2^{-}$ & At the interface & $9^{+} 10^{-}$ & Outside \\
\hline DBS 02 & $1^{-} 2^{+}$ & At the interface & $9^{-} 10^{+}$ & At the interface \\
\hline DBS 03 & $0^{-} 3^{+}$ & Outside & $9^{-} 11^{+}$ & Outside \\
\hline DBS 04 & $1^{-} 3^{+}$ & At the interface & $10^{-} 11^{+}$ & $8^{+} 11^{-}$ \\
\hline DBS 05 & $\mathrm{C}^{+} 2^{-}$ & At the interface & $8^{-} 11^{+}$ & At the interface \\
\hline DBS 06 & $1^{-} 3^{+}$ & Within & $\mathrm{C}^{+} 10^{-}$ & At the interface \\
\hline DBS 07 & $\mathrm{C}^{+} 2^{-}$ & $9^{-} 10^{+}$ & Outside \\
\hline DBS 08 & $1^{-} 2^{+}$ & $9^{-} 11^{+}$ & At the interface \\
\hline DBS 09 & $\mathrm{C}^{+} 2^{-}$ & Outside & $9^{-} 10^{+}$ & Outside \\
\hline DBS 10 & $1^{-} 2^{+}$ & Outside & Outside & \\
\hline
\end{tabular}

Electrode contact localization was performed using the Lead-DBS toolbox in Matlab (Mathworks, Inc., Natick, MA). ${ }^{25}$ The post-operative MRI was linearly co-registered with the pre-operative MRI. The co-registered acquisitions were non-linearly normalized into MNI space using the DARTEL method implemented using SPM12. ${ }^{26}$ Coordinates of the active contacts were acquired and each contact was classified as either "within" or "outside" the STN, or "at the interface" between the STN and overlying structures. ${ }^{27}$ Two authors (AA and GG) independently assessed the anatomical position of each contact in relation to the STN in the coronal, sagittal, and transverse planes. Inter-rater reliability for contact coordinates within AC-PC stereotactic space ranged from $98.3 \%$ to $99.9 \%$. ANOVA revealed no significant effect on any of the speech measures based on anatomical contact location. 
of the 5 visits). Speech was recorded using a unidirectional condenser headset microphone (DPA 4060, $6 \mathrm{~cm}$ from the mouth) attached to a portable digital audio recorder (M-Audio Microtrack 2). The following two tasks were analyzed: (1) prolonged vowel "ah" and (2) sentence production of "She Saw Patty Buy Two Poppies." Measures of speech intensity (dB), voice quality (jitter \% or cycle-to-cycle frequency variation and shimmer $\%$ or cycle-to-cycle amplitude variation values, harmonics-noise/ $(H / N)$ ratio which is a reflection of the amount of additive noise in the voice signal), and speech prosody acoustics (semitone standard deviation (STSD) with higher relative values in this measure indicating improved prosodic variation) were obtained using the software program Praat. ${ }^{28}$ Average speech intensity was obtained with the root mean squared (RMS) intensity contour method from both a 2-s mid-section of the prolonged vowel and from the entire sentence production with long $(+500 \mathrm{~ms})$ unvoiced segments or pauses selectively removed. Voice quality measures were obtained from a 2-s mid-section of the prolonged vowel and STSD from the duration of the sentence production. STSD was calculated from the mean and standard deviation of fundamental frequency $\left(F_{0}\right)$ in hertz using the following equation: $\mathrm{STSD}=12 / 0.301 \times \log [(\mathrm{Hz}$ mean $+\mathrm{Hz} \mathrm{SD} / 2) /(\mathrm{Hz}$ mean $-\mathrm{Hz} \mathrm{SD} / 2)]$. Listener ratings (5 speech language pathology graduate students) of voice quality and prosody (visual analog rating scales) were obtained from the vowel and sentence audio-recordings, respectively, for each STN-DBS condition. Listeners heard and evaluated each sample twice and average ratings were used for the analysis. The Pearson correlation coefficients obtained for intra-rater and inter-rater reliability were 0.86 and 0.83 , respectively.

\section{Analysis}

All data were tested for normality using the Shapiro-Wilk normality test. The tests were not significant, and therefore, normality was assumed for all subsequent analyses.

\section{Non-experimental Comparisons}

Average data across participants were used for all of the following analyses. Visit 1 (pre-operative) was compared to Visit 2 (post-operative, DBS “off”) to examine any potential microlesion effects from the surgical procedure itself. ${ }^{29}$ Visit 1 was also compared to Visit 3 (low-voltage DBS) to examine potential effects of low-voltage setting stimulation for speech when there is no other observable motor benefit. Visit 4 was compared to the final clinical setting to determine the effect of the stimulator settings from initial programming to final clinical programming.

\section{Selection of Optimal Settings for Speech}

Settings for each of the three electrical parameters (frequency, voltage, and pulse width) were binned into three categories: low, mid, and high. This binning procedure was selected to assess the relative contribution of each setting in order to simplify potential clinical recommendations. Parameter setting bins are reported in Table 3. The effects on speech at Visits 3-7 (experimental sessions) compared to Visit 8 (standard clinical setting, 6-month post-operative; selected by the neurologist to address cardinal motor symptoms) were examined to determine the effect of STN-DBS parameter permutations and optimization potential for speech. The optimal setting for each speech measure (jitter, $H / N$,
Table 3: Parameter setting binning

\begin{tabular}{l|c|c|c}
\hline & Frequency $(\mathbf{H z})$ & Pulse width $(\mu \mathbf{s})$ & Voltage $(\mathbf{V})$ \\
\hline Low & $60-90$ & $60-90$ & $1-2$ \\
\hline Mid & $120-130$ & $130-150$ & $3-4$ \\
\hline High & 180 & 210 & 4.5 \\
\hline
\end{tabular}

shimmer, STSD, intensity, perceptual ratings of voice quality, and prosody) was first compared to the standard clinical DBS settings. The median values for the standard clinical settings were $130 \mathrm{~Hz}$, $120 \mu \mathrm{s}$, and $3.4 \mathrm{~V}$. The standard clinical settings were also compared with the optimal speech settings for each participant (not all participants were able to tolerate each parameter setting tested; as such, if a participant found an experimental setting uncomfortable, the session was terminated and replaced with the following randomly selected setting. Thus, not all participants underwent the same number of optimal setting combinations). Optimal speech settings were identified by first examining each parameter setting in isolation. The optimal frequency of stimulation was determined by comparing results (paired $t$-tests) from each speech measure across participants for low, mid, and high frequency to the standard clinical setting. The same analysis was completed for pulse width and voltage. All possible combinations of these optimal settings were then analyzed to account for the combined parameters typically adjusted in STN-DBS. These were referred to as the optimal combined settings. Paired, two-tailed $t$-tests were used to determine whether each of these optimal combined settings led to significant improvements in each of the speech measures compared to the standard clinical setting.

Corrections for multiple comparisons in the current study were not completed due to concerns related to Type 2 errors. We present data as preliminary and exploratory, and replications in the future should involve statistical procedures for multiple comparison corrections and larger sample sizes.

\section{Total Electrical Energy Delivered}

Total Electrical Energy Delivered (TEED) delivered to the STN was calculated as TEED $_{1 \mathrm{~s}}=\left[\left(\right.\right.$ voltage $^{2} \times$ frequency $\times$ pulse width)/impedance] $\times 1 \mathrm{~s} .{ }^{30}$ Pearson correlation analysis was used to examine the relationship between TEED and each of the speech measures.

\section{RESUlTS}

Ten individuals with PD (mean age, 63.9 years; range, 52-69 years) who received bilateral STN-DBS participated in the current study. Mean years since diagnosis was 10.6 years (range, 6-17 years). Participants presented with no or only mild dysarthria symptoms at baseline and no other speech-related complaints.

\section{Non-experimental Comparisons}

The post-operative DBS off condition (Visit 2) was found to have a significantly reduced STSD $(2.79 \pm 1.61)$ compared to the pre-operative condition (Visit 1) $(3.51 \pm 1.84) \quad(t(9)=2.77$, $p=0.022$ ). The post-operative DBS off condition was also observed to have a reduced vowel intensity $(67.97 \pm 3.49)$ compared to the pre-operative vowel intensity $(70.58 \pm 4.51)(t(9)=2.553$, 
$p=0.031$ ). This suggests that either there was a detrimental surgical lesion effect on speech prosody and speech intensity or there was a pharmacological impact on these speech symptoms, or a combination of both was observed. The initial clinician-based DBS programming (Visit 4) was found to have significantly higher STSD $(4.38 \pm 2.07)$ compared to the Visit 2 (post-operative, DBS off) STSD measure $(2.90 \pm 1.67)(t(8)=2.58, p=0.032)$, suggesting that although either micro-lesion effects from surgery or lack of PD medication had a negative impact on speech prosody, this was resolved through low-level stimulation. Similarly, increased sentence intensity at Visit 4 (initial programming session) $(66.62 \pm 2.29)$ was observed compared to Visit 2 (post-operative, DBS off) $(64.61 \pm 2.51)$ and at Visit 3 (post-operative, DBS on, minimal stimulation) $(63.75 \pm 2.48)(t(8)=-2.941, p=0.019$; $t(8)=-5.25, p=0.001$, respectively). Interestingly, we found reduced STSD at Visit 8 (final clinical setting) $(2.41 \pm 1.45$ ) compared to Visit 4 (initial programming) $(4.38 \pm 2.07)(t(8)$ $=2.31, p=0.050$ ), suggesting a decline in speech prosody by the end of STN-DBS programming. We did not find any other differences in the non-experimental comparisons. These comparisons suggest that vowel intensity and the prosodic measure of pitch variability may be sensitive measures of speech change following STN-DBS surgery. In addition, these comparisons highlight the minimal change in speech intensity, voice quality, and speech prosody from pre-surgical levels to post-surgical clinical settings. This is in contrast to the numerous significant effects found for the optimal experimental conditions explored below.

\section{Optimal Speech Settings}

The best speech score for each of the measures (jitter, $H / N$, shimmer, STSD, intensity, perceptual ratings of voice quality, and prosody) was compared to the standard clinical DBS settings to explore the potential for STN-DBS optimization. Paired $t$-test analysis revealed significant improvement with the best speech score from experimental conditions compared to the standard clinical setting for all of the speech measures $(p<0.05)$ (refer to Table 4).

\section{Frequency of STN-DBS Stimulation}

Mid frequency led to improved $H / N(19.98 \pm 3.52)$ compared to the standard clinical setting $(18.77 \pm 4.85)$; however, this only approached significance $(t(9)=1.99, p=0.078)$. Low $(3.70 \pm 0.92)$ and mid frequency $(3.33 \pm 0.58)$ led to improved STSD compared to the standard clinical setting $(2.37 \pm 1.37)$ $(t(9)=2.70, p=0.024 ; t(9)=2.47, p=0.036$, respectively). Low $(66.30 \pm 2.22)$ and mid frequency $(67.48 \pm 2.49)$ also led to improved sentence intensity compared to the standard clinical setting $(64.55 \pm 3.00)(t(9)=2.51, p=0.033 ; t(9)$ $=4.11, p=0.03$, respectively).

\section{Pulse Width of STN-DBS Stimulation}

Mid pulse width led to improved $H / N(20.28 \pm 3.37)$ compared to the standard clinical setting $(18.77 \pm 4.85)$; however, this only approached significance $(t(9)=2.1, p=0.065)$. Low pulse width led to improved STSD $(3.35 \pm 0.89)$ compared to the standard clinical setting $(2.37 \pm 1.37)(t(9)=2.40, p=0.040)$, and improvement with mid pulse width $(3.64 \pm 1.14)$ approached significance $(t(9)=2.08, p=0.067)$. Low $(67.12 \pm 1.96)$ and mid pulse width $(66.39 \pm 3.01)$ led to improved sentence intensity
Table 4: Means for each speech outcome measure under standard clinical settings and the best speech scores. $p$ values obtained from paired $t$-tests for each speech out- come comparing the standard clinical setting and the best speech score

\begin{tabular}{l|c|c|c}
\hline $\begin{array}{l}\text { Speech } \\
\text { measure }\end{array}$ & $\begin{array}{c}\text { Best speech } \\
\text { score }\end{array}$ & $\begin{array}{c}\text { Standard } \\
\text { clinical setting }\end{array}$ & $\boldsymbol{p}$ \\
\hline Jitter (\%) & 0.270 & 0.603 & 0.016 \\
\hline$H / N$ ratio & 24.462 & 18.770 & 0.000 \\
\hline Shimmer (\%) & 2.567 & 6.118 & 0.007 \\
\hline STSD & 8.33 & 2.37 & 0.001 \\
\hline $\begin{array}{c}\text { Vowel } \\
\text { intensity (dB) }\end{array}$ & 77.700 & 63.653 & 0.026 \\
\hline $\begin{array}{c}\text { Sentence } \\
\text { intensity (dB) }\end{array}$ & 73.188 & 64.552 & 0.000 \\
\hline $\begin{array}{c}\text { Perceptual rating of } \\
\text { voice quality }(\%)\end{array}$ & 48.0 & 30.3 & 0.012 \\
\hline $\begin{array}{c}\text { Perceptual rating of } \\
\text { pitch variability } \\
(\%)\end{array}$ & 59.3 & 30.1 & \\
\hline
\end{tabular}

compared to the standard clinical setting $(64.55 \pm 3.00)(t(9)$ $=3.53, p=0.006 ; t(9)=2.26, p=0.050$, respectively).

\section{Voltage of STN-DBS Stimulation}

Mid voltage led to improved STSD $(3.47 \pm 0.56)$ compared to the standard clinical setting $(2.37 \pm 1.37)(t(9)=2.71, p=0.024)$. Low $(67.04 \pm 2.12)$ and mid voltage $(67.11 \pm 2.43)$ led to improved sentence intensity compared to the standard clinical setting $\quad(64.55 \pm 3.00) \quad(t(9)=3.38, \quad p=0.008 ; \quad t(9)=3.19$, $p=0.011$, respectively), with high-voltage improvements $(66.17 \pm 2.31)$ approaching significance $(t(9)=2.13, p=0.062)$.

We did not find any other significant comparisons in other speech measures. The results suggest that, in general, low-mid frequency, low-mid pulse width, and mid-high voltage were associated with improvements in the speech measures, compared to the standard clinical settings.

All possible combinations (eight combinations; refer to Table 5.) of these optimal settings were then tested, and paired $t$-tests revealed that three specific combinations of settings were associated with improved speech scores compared to the standard clinical settings $(3=$ low frequency, mid pulse width, mid voltage; 4 = low frequency, mid pulse width, high voltage; $5=$ mid frequency, low pulse width, mid voltage; refer to Table 6). Harmonics-noise $(21.52 \pm 2.65)$ was associated with improved scores with optimal setting no. 3 (low frequency, mid pulse width, and mid voltage) compared to the standard clinical setting $(18.33 \pm 4.93)(H / N, t(8)=2.550, p=0.034)$, with shimmer $(4.32 \pm 1.16)$ and perceptual ratings of pitch variability $(4.6 \pm 0.94)$ comparisons to the standard clinical settings $(6.55 \pm 3.97 ; 3.01 \pm 1.62$, respectively) approaching significance for this setting ( $p=0.06 ; p=0.08$, respectively). Improved speech intensity (vowel, 72.80 \pm 3.44 ; sentence, $67.40 \pm 2.45)$ was associated with optimal setting no. 4 (low frequency, mid pulse width, and high voltage) compared 
Table 5: All possible combinations of identified optimal settings for speech

\begin{tabular}{l|c|c|c}
\hline $\begin{array}{l}\text { Optimal } \\
\text { combination no. }\end{array}$ & Frequency & Pulse width & Voltage \\
\hline 1 & Low & Low & Mid \\
\hline 2 & Low & Low & High \\
\hline 3 & Low & Mid & Mid \\
\hline 4 & Low & Mid & High \\
\hline 5 & Mid & Low & Mid \\
\hline 6 & Mid & Low & High \\
\hline 7 & Mid & Mid & Mid \\
\hline 8 & Mid & Mid & High \\
\hline
\end{tabular}

to the standard clinical setting (vowel, $67.61 \pm 4.37, t(6)=4.984$, $p=0.002$; sentence intensity, $64.11 \pm 3.30, t(6)=2.584, p=$ $0.042)$, with improved shimmer $(3.74 \pm .91)$ and harmonicsnoise $(20.5 \pm 2.86)$ compared to the standard clinical setting $(6.12 \pm 3.99 ; 17.21 \pm 4.75$, respectively) approaching significance $(p=0.06)$. Improved STSD $(3.04 \pm 1.20)$ and sentence intensity $(67.9 \pm 2.51)$ were associated with improvements with optimal setting no. 5 (mid frequency, low pulse width, and mid voltage) compared to the standard clinical setting $(2.37 \pm 1.37 ; 64.55 \pm 3.0$, respectively) (STSD, $t(9)=3.06, p=0.014$; sentence intensity, $t(9)=6.471, p=0.000)$, with harmonics-noise $(20.35 \pm 4.33)$ compared to the standard clinical setting $(18.77 \pm 4.85)$ approaching significance $(p=0.054)$. Significant comparisons were not found for jitter, shimmer, or perceptual ratings of voice quality measures. It is important to note that the mean standard clinical setting was equivalent to optimal setting no. 1 (low frequency, low pulse width, mid voltage); however, this setting was not related to specific improvements in any of the speech measures evaluated.

\section{Total Electrical Energy Delivered}

Pearson correlation analysis of the relationship between TEED and voice quality measures revealed weak correlations $(r=0.162, p<0.05 ; r=0.186, p<0.01 ; r=-0.277, p<0.01)$ for jitter, shimmer, and $H / N$, respectively. Additionally, weak negative correlations were found between TEED and the speech measures of vowel intensity $(r=-0.175, p<0.05)$, and sentence intensity $(r=-0.157, p<0.05)$.

\section{Discussion}

\section{Limitations}

Several limitations of the current study are identified. The small sample size of 10 individuals and the large amount of individual variability across STN-DBS settings warrant caution in clinical application of the suggested parameter settings. Second, transient speech effects of the stimulation settings are possible and long-term investigation of the stability of observed speech outcomes is suggested. Furthermore, the current study did not include limb or other axial symptom comparisons. This limitation impacts our understanding of the overall impact of optimization for speech and careful consideration of patient symptomology is recommended during programming. Finally, many patients in the current study received bipolar stimulation; only few receiving monopolar stimulation (refer to Table 2) were able to tolerate the treatment settings. It is important to consider the type of stimulation when programming and caution is recommended as the current study findings relate primarily to a bipolar mode of stimulation. Future research is recommended in the area of monopolar stimulation related to different parameter settings.

\section{Summary}

The current study explored performance on a number of speech measures across a range of stimulation parameter manipulations. In the current study, we found that standard clinical settings are not optimized for speech. Results from 10 individuals with PD show that improvements in mean speech intensity, jitter, shimmer, $H / N$ ratio, STSD, and perceptual ratings of voice quality and pitch variability were associated with the following stimulation parameters: lower frequency $(60-130 \mathrm{~Hz})$, lower pulse width $(60-150 \mu \mathrm{s})$, and higher voltage (3-4.5 V). These parameter combinations appear to be associated with improved

Table 6: Means (and standard deviation) for each speech outcome measure and the best three optimal setting combinations

\begin{tabular}{|c|c|c|c|}
\hline Speech measure & $\begin{array}{c}\text { Optimal setting no. } 3 \\
\text { (low frequency, mid } \\
\text { pulse width, mid voltage) }\end{array}$ & $\begin{array}{l}\text { Optimal setting no. } 4 \\
\text { (low frequency, mid pulse } \\
\text { width, high voltage) }\end{array}$ & $\begin{array}{c}\text { Optimal setting no. } 5 \\
\text { (mid frequency, low } \\
\text { pulse width, mid voltage) }\end{array}$ \\
\hline Jitter $(\%)$ & $0.45(0.11)$ & $0.47(0.17)$ & $0.56(0.35)$ \\
\hline$H / N$ ratio & $21.52(2.65)^{*}$ & $20.5(2.86)$ & $20.35(4.33)$ \\
\hline Shimmer $(\%)$ & $4.32(1.16)$ & $3.74(0.91)$ & $5.54(3.79)$ \\
\hline STSD & $3.71(2.30)$ & $3.12(1.60)$ & $3.04(1.20)^{*}$ \\
\hline Vowel intensity (dB) & $72.56(4.56)$ & $72.80(3.44)^{*}$ & $72.46(3.08)$ \\
\hline Sentence intensity $(\mathrm{dB})$ & $65.29(4.86)$ & $67.40(2.45)^{*}$ & $67.89(2.51)^{* *}$ \\
\hline Perceptual rating of voice quality (\%) & $33(8.0)$ & $25(12.6)$ & $41(13.8)$ \\
\hline Perceptual rating of pitch variability $(\%)$ & $46(9.4)$ & $28(22)$ & $48(7.2)$ \\
\hline
\end{tabular}

*Indicates significant difference with the standard clinical setting $<0.05$;

**Indicates significant difference with the standard clinical setting $<0.000$. 
speech outcomes compared to standard clinical settings. The mean values for the standard clinical settings were $117 \mathrm{~Hz}$ (low), $95 \mu \mathrm{s}$ (low), and 3.4 Volts (mid). Despite the approximation of the standard clinical settings to the optimal settings identified in the current study, the specific combination of low frequency, low pulse width, and mid voltage did not result in improved speech measures in our participants. Therefore, although these results highlight the potential optimization of STN-DBS for speech, they also highlight the complex interaction of the parameter settings when programming the device. A starting point from which exploration of STN-DBS clinical programming may begin includes addressing improved intensity of speech with higher voltage, lower frequency, and mid pulse width. Prosodic concerns may be addressed by exploring mid voltage with mid frequency and lower pulse width. Voice quality concerns could be explored using mid voltage with low frequency and mid pulse width.

Due to variability between patients, speech measures, and low sample size in the current study, these optimization recommendations may not be appropriate for all patients but rather provide a starting point or guideline from which to program DBS devices. Individuals with PD may present with one or multiple pre-morbid speech-related symptoms. Existing concerns may be identified in collaboration with speech-language pathology professional. These concerns may be addressed depending on the relative impact of each speech symptom on the patient's daily life and communication ability with family and friends. Clinical judgment may be used to determine the optimal setting for each participant; however, to date, there is limited literature support for exploration of all three STN-DBS settings from which neurologists can begin to treat speech symptoms.

It is important to acknowledge the complex interaction between stimulation parameters, positioning of electrodes within the anatomical structures, and the contact positioning of each electrode for optimal clinical benefit. ${ }^{31}$ Active electrode contacts were localized, and we are able to confirm targets being within, outside, or on the border of the STN. Previous researchers highlight methodological concerns related to electrode contact localization procedures and although we did not find differences in speech outcome measures related to active electrode contact location, future research is required to understand the relationship between anatomical location of contacts and its impact on clinical benefit. $^{27}$

Some studies report involvement of the dentatorubrothalamic tract (DRTt) in dysarthria following STN-DBS surgery. ${ }^{32,33}$ The DRTt efferent projections connect the dentate nucleus in the cerebellum (deep nuclei), the red nucleus, and the thalamus. ${ }^{34}$ Fenoy et al. ${ }^{33}$ suggest that reduced perceptual ratings of voice quality and prosody found in their study were related to current diffusion to the DRTt. Other studies cite the impact of current diffusion to corticobulbar fibers as the most probable cause of slurred speech and intensity worsening, as this can lead to contractions of facial, laryngeal, or respiratory muscles. ${ }^{6,35}$ The current findings are not discordant with this hypothesis. Increased frequency of stimulation, which results in the increased spread of neuronal stimulation, indeed led to worsened speech intensity, voice quality, and prosodic control. Increased stimulation settings (above that which is optimal for cardinal PD symptoms) have been associated with reduced speech intensity in another study as well. ${ }^{6}$
Positive correlations were found for TEED with jitter and shimmer. This suggests that with decreased electrical current delivered to the STN, irrespective of the specific stimulator parameter settings, there was a decrease in the amount of abnormal vibration patterns of the voice in individuals with PD (increased vocal stability). Further, negative correlational relationships were found between TEED and $H / N$ and speech intensity. Therefore with decreased electrical current, there was a pattern of increased speech intensity and improved distinctiveness of the speech sound harmonics. This is consistent with previous work suggestive of speech deterioration with increased TEED as evaluated using the UPDRS speech rating. ${ }^{17}$ This group of researchers found that higher UPDRS speech scores (worsened speech) were associated with increased TEED to the right STN compared to the left STN. ${ }^{17}$ These results are inconsistent with previous work on variable TEED (TEED that is not held constant) for cardinal symptom alleviation. Previous research suggests that increased TEED leads to improvements in most motor symptoms such as bradykinesia, rigidity, and tremor. ${ }^{36}$

It is unclear whether pre-morbid speech indices are predictive of STN-DBS outcome. Klostermann et al. ${ }^{8}$ included subjects with worse pre-operative voice quality and found resistance to change following stimulation. This is in contrast to Gentil et al. ${ }^{12}$ who found improved voice quality in their participants with relatively stable voice quality with stimulation "off." Few studies have examined pre-morbid conditions in relation to stimulator settings. This is an important consideration for future research as participants in the current study were identified as having no or mild dysarthria symptoms.

The results of the current study were obtained from standard measures of speech intensity, voice quality, and prosody. Future studies should include other indices of voice quality and intensity valid for PD-related dysarthria such as tremor, subharmonic component, voice irregularity-related analyses, intensity declination, and intensity variability.

In the current study, optimized speech settings did not align with the standard clinical settings used to address the major motor symptoms of PD. Thus, the authors suggest careful clinical judgment when determining STN-DBS programming based on relative importance of addressing major motor symptoms and speech symptoms. Considerations may include different preprogrammed setting combinations depending on the patient's daily needs, for example, spending time sitting while conversing at home or walking in the grocery store.

\section{ACKNOWLedgements}

This research was supported by funding awarded to GG from the Canadian Institutes of Health Research.

\section{Author Contributions}

AA designed and conceptualized study; analyzed and interpreted data; and drafted manuscript for intellectual content. SA designed and conceptualized study; analyzed and interpreted data; and revised manuscript for intellectual content. CM revised manuscript for intellectual content. TK revised manuscript for intellectual content. GG designed and conceptualized study; analyzed data; and revised manuscript for intellectual content. MD designed and conceptualized study. MJ designed and conceptualized study. 


\section{DisClosures}

GG reports a grant from Canadian Institute for Health Research, during the conduct of the study. SA received salary support from Western University and received research grants from Parkinson Canada, Parkinson Society of Southwestern Ontario, and Merz Pharma. AA was a graduate student at Western University and received a graduate student award from Parkinson Canada. TK was a graduate student at Western University and received a graduate student award from the Parkinson Society of Southwestern Ontario. MJ has received grants from CIHR, MITACS, OCE, Merz, Allergan, Abbvie and Boston Scientific. MJ has also received speaker fees from Merz, Allergan, Abbvie and UCB Pharma. MD and CM have nothing to disclose.

\section{REFERENCES}

1. Duffy JR. Motor speech disorders: substrates, differential diagnosis, and management, 3rd ed. St. Louis, MO: Elsevier, Mosby; 2013.

2. Tewari A, Jog R, Jog M. The striatum and subthalamic nucleus as independent and collaborative structures in motor control. Front Syst Neurosci. 2016;10:17.

3. Benabid AL, Chabardes S, Mitrofanis J, Pollak P. Deep brain stimulation of the subthalamic nucleus for the treatment of Parkinson's disease. Lancet Neurol. 2009;8(1):67-81.

4. Kern DS, Kumar R. Deep brain stimulation. Neurologist. 2007; 13(5):237-52.

5. Mazzone P, Lozano A, Stanzione P, et al. Implantation of human pedunculopontine nucleus: a safe and clinically relevant target in Parkinson's disease. Neuroreport. 2005;16:1877-81.

6. Pinto S, Gentil M, Krack P, et al. Changes induced by levodopa and subthalamic nucleus stimulation on Parkinsonian speech. Mov Disord. 2005;20:1507-15.

7. Moreau C, Pennel-Ployart O, Pinto S, et al. Modulation of dysarthropneumophonia by low-frequency STN DBS in advanced Parkinson's disease. Mov Disord. 2011;26(4):659-63.

8. Sidiropoulos C, Walsh R, Meaney C, Poon YY, Fallis M, Moro E. Low-frequency subthalamic nucleus deep brain stimulation for axial symptoms in advanced Parkinson's disease. J Neurol. 2013;260(9):2306-11.

9. Klostermann F, Ehlen F, Vesper J, et al. Effects of subthalamic deep brain stimulation on dysarthrophonia in Parkinson's disease. J Neurol Neurosurg Psychiatr. 2008;79:522-9.

10. D'Alatri L, Paludetti G, Contarino MF, Galla S, Marchese MR, Bentivoglio AR. Effects of bilateral subthalamic nucleus stimulation and medication on Parkinsonian speech impairment. J Voice. 2008;22:365-72.

11. Dromey C, Kumar R, Lang A, Lozano A. An investigation of the effects of subthalamic nucleus stimulation on acoustic measures of voice. Mov Disord. 2000;15:1132-8.

12. Gentil M, Pinto S, Pollak P, et al. Effect of bilateral stimulation of the subthalamic nucleus on Parkinsonian dysarthria. Brain Lang. 2003:85:190-6.

13. Goetz CG, Fahn S, Martinez-Martin P, et al. Movement Disorder Society-sponsored revision of the Unified Parkinson's Disease Rating Scale (MDS-UPDRS): process, format, and clinimetric testing plan. Mov Disord. 2007;22(1):41-7.

14. Krause M, Fogel W, Mayer P, Kloss M, Tronnier V. Chronic inhibition of the subthalamic nucleus in Parkinson's disease. J Neurol Sci. 2004;219:119-24.

15. Mate MA, Cobeta I, Jiménez-Jiménez FJ, Figueiras R. Digital voice analysis in patients with advanced Parkinson's disease undergoing deep brain stimulation therapy. J Voice. 2012;26:496-501.

16. Santens P, De Letter M, Van Borsel J, De Reuck J, Caemaert J. Lateralized effects of subthalamic nucleus stimulation on different aspects of speech in Parkinson's disease. Brain Lang. 2003;87:253-58.

17. Skodda S, Gronheit W, Schlegel U, Sudmeyer M, Schnitzler A, Wojtecki L. Effect of subthalamic stimulation on voice and speech in Parkinson's disease: for the better or worse? Front Neurol. 2014;4:218.

18. Tanaka Y, Tsuboi T, Watanabe H, et al. Voice features of Parkinson's disease patients with subthalamic nucleus deep brain stimulation. J Neurol. 2015;262:1173-81.

19. Tripoliti E, Zrinzo L, Martinez-Torres I, et al. Effects of contact location and voltage amplitude on speech and movement in bilateral subthalamic nucleus deep brain stimulation. Mov Disord. 2008;23:2377-83.

20. Valalik I, Smehak G, Bognar L, Csokay A. Voice acoustic changes during bilateral subthalamic stimulation in patients with Parkinson's disease. Clin Neurol Neurosurg. 2011;113: $188-95$

21. Xie Y, Zhang Y, Zheng Z, et al. Changes in speech characters of patients with Parkinson's disease after bilateral subthalamic nucleus stimulation. J Voice. 2011; 25:751-8.

22. Isaias IU, Tagliati M. Deep brain stimulation programming for movement disorders. In: Tarsy D, Vitek JL, Starr P, Okun M, editors. Current clinical neurology: deep brain stimulation in neurological and psychiatric disorders. Totowa, NJ: Springer; 2008, pp. 361-97.

23. Groiss SJ, Wojtecki L, Südmeyer M, Schnitzler A. Deep brain stimulation in Parkinson's disease. Ther Adv Neurol Disord. 2009;2(6):20-8.

24. Tomlinson CL, Stowe R, Patel S, Rick C, Gray R, Clarke CE. Systematic review of levodopa dose equivalency reporting in Parkinson's disease. Mov Disord. 2010;25(15):2649-53.

25. Horn A, Kühn A. Lead-DBS: a toolbox for deep brain stimulation electrode localizations and visualizations. NeuroImage. 2014; 107:127-35.

26. Ashburner J. A fast diffeomorphic image registration algorithm. Neuroimage. 2007;38:95-113.

27. Claire F, Ranoux D, Guehl D, Burbaud P, Cuny E. A systematic review of studies on anatomical position of electrode contacts used for chronic subthalamic stimulation in Parkinson's disease. Acta Neurochirurg. 2013;155:1647-54.

28. Boersma P, Weenink D. PRAAT (version 5.0.20) [Software]; 2008. Available at: http://www.fon.hum.uva.nl/praat/.

29. Jech R, Mueller K, Urgosik D, et al. The subthalamic microlesion story in Parkinson's disease: electrode insertion-related motor improvement with relative cortico-subcortical hypoactivation in fMRI. PLoS ONE. 2012;7(11):e49056.

30. Koss AM, Alterman RL, Tagliati M, Shils JL. Calculating total electrical energy delivered by deep brain stimulation systems. Ann Neurol. 2005;58:168.

31. di Biase L, Fasano A. Low-frequency deep brain stimulation for Parkinson's disease: great expectation or false hope? Mov Disord. 2016;31:962-7.

32. Tommasi G, Krack P, Fraix V, et al. Pyramidal tract side effects induced by deep brain stimulation of the subthalamic nucleus. J Neurol Neurosurg Psychiatr. 2008;79:813-19.

33. Fenoy AJ, McHenry MA, Schiess MC. Speech changes induced by deep brain stimulation of the subthalamic nucleus in Parkinson disease: involvement of the dentatorubrothalamic tract. J Neurosurg. 2016;126(6):2017-27.

34. Carpenter MB. Core text of neuroanatomy. Baltimore: Williams and Wilkins; 1991.

35. Kumar R, Johnson L. Managing Parkinson's disease patients treated with deep brain stimulation. In: Marks WJ, editor. Deep brain stimulation management. New York: Cambridge University Press; 2011.

36. Moro E, Esselink RJA, Xie J, et al. The impact on Parkinson's disease of electrical parameter settings in subthalamic nucleus stimulation. Neurology. 2002;59:706-713. 